\title{
JEM-X observations of the Be/X-ray binary EXO 2030+375^
}

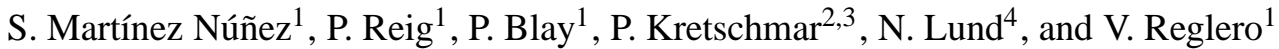 \\ 1 GACE, Instituto de Ciencias de los Materiales, Universidad de Valencia, PO Box 20085, 46071 Valencia, Spain \\ 2 Max-Planck-Institut für extraterrestrische Physik, Giessenbachstrasse, 85748 Garching, Germany \\ 3 INTEGRAL Science Data Centre, Chemin d'Écogia 16, 1290 Versoix, Switzerland \\ ${ }^{4}$ Danish Space Research Institute, Juliane Maries Vej 30, 2100, Copenhagen, Denmark
}

Received 7 July 2003 / Accepted 1 August 2003

\begin{abstract}
We have used data from the Joint European Monitor (JEM-X) to perform an X-ray spectral and timing analysis of the 42-s transient pulsar EXO 2030+375 during an X-ray outburst. X-ray pulsations are clearly detected with an average pulse period of $41.66 \pm 0.05 \mathrm{~s}$ and an average pulse fraction of $60 \%$. The profile of the energy spectrum did not change appreciably throughout the X-ray outburst, although the source shows a slightly softer spectrum during periastron passage in the energy range 9-25 keV. The 5-25 keV X-ray luminosity changed by a factor of 2 throughout the observations, reaching a maximum value of $3 \times 10^{36} \mathrm{erg} \mathrm{s}^{-1}$. These observations allowed us to verify the in-flight instrumental properties of the JEM-X Monitor.
\end{abstract}

Key words. INTEGRAL - JEM-X- BeX stars - HMXRBs - EXO 2030+375

\section{Introduction}

EXO 2030+375 belongs to the subclass of high-mass X-ray binaries known as Be/X-ray binary (Coe 2000 and references therein). In these systems a neutron star orbits around a Be star. Be stars are rapidly rotating B-type stars that show emission lines and infrared excess when compared to normal B-type stars. The line emission and the infrared excess are believed to be associated with a quasi-Keplerian disk formed by material flowing out from the Be star (Balona 2000 and references therein). The X-ray outbursts are produced when the neutron star accretes matter from the circumstellar disk.

When active, the X-ray flux of EXO 2030+375 is modulated by two types of periodic phenomena. 46-day outbursts are detected during periastron passage of the compact star (Wilson et al. 2002). These outbursts are known as type I outbursts and are characterised by moderate increases of the X-ray luminosity $\left(L_{x}<10^{37} \mathrm{erg} \mathrm{s}^{-1}\right)$, low spin-up rates (if any) and recurrence at the orbital period. In addition to this long-term variation, the X-ray flux exhibits 41.7-s pulsations (Parmar et al. 1989; Reynolds et al. 1993; Reig \& Coe 1998a), which are attributed to the rotation of the neutron star and results from the fact that the magnetic and rotation axis are misaligned.

Send offprint requests to: S. Martínez Núñez, e-mail: silvia.martinez@uv.es

* Based on observations with INTEGRAL, an ESA project with instruments and science data centre funded by ESA member states (especially the PI countries: Denmark, France, Germany, Italy, Switzerland, Spain), Czec Republic and Poland, and with the participation of Russia and the USA.
Occasionally, Be/X-ray binaries also show giant outbursts (known as type II outbursts). During these outbursts the X-ray luminosity reaches near Eddington values $\left(L_{x} \sim 10^{38} \mathrm{erg} \mathrm{s}^{-1}\right.$ for a neutron star). They do not correlate with the orbital phase and high spin-up rates are observed. For EXO 2030+375, only one giant outburst - the one that led to its discovery - has been so far reported (Parmar et al. 1989).

In common with other high mass X-ray binaries, the continuum spectral shape of EXO $2030+375$ in the range 2-20 keV has been represented by a power law function $(\Gamma=1.00 \pm$ $0.06)$, an exponential cut-off $(\sim 8.85 \pm 0.33 \mathrm{keV})$, coupled with a low-energy absorption $\left(N_{\mathrm{H}}=2.6 \pm 0.3 \times 10^{22} \mathrm{~cm}^{-2}\right)$ and an iron emission line at $\sim 6.5 \mathrm{keV}$ (Reig \& Coe 1999).

The optical counterpart to EXO $2030+375$ is a relatively faint $(\mathrm{V}=20)$ BO Ve star (Motch \& Jacot-Pacheco 1987; Coe et al. 1988), showing long-term optical and infrared variability (Reig et al. 1998b). The orbital parameters of the system can be found in Table 2 of Wilson et al. (2002).

In this paper, we have performed an X-ray timing and spectral analysis of EXO 2030+375 during a type I outburst, using data from the Joint European Monitor (JEM-X) on-board INTEGRAL.

\section{Observations and data reduction}

The data that have been analysed in this work were obtained during the INTEGRAL Performance and Verification phase (PV), which lasted from launch on October 17, 2002 to the end of December 2002. During this phase INTEGRAL was observing mostly the Cygnus $\mathrm{X}-1$ region, performing 
Table 1. EXO 2030+375 journal of observations.

\begin{tabular}{cccccccc}
\hline \hline Obs. number & SWID & IJD & Obs. time $(\mathrm{s})$ & $S / N$ & Orbital phase & counts/s & $C L$ \\
\hline 1 & 001900100010 & 1073.61300 & 1800 & 23.6 & 0.9876 & $3.17 \pm 0.17$ & $>99 \%$ \\
2 & 001900110010 & 1073.63503 & 1798 & 13.0 & 0.9881 & $1.96 \pm 0.15$ & - \\
3 & 001900400010 & 1074.27360 & 1796 & 29.1 & 0.0020 & $4.19 \pm 0.18$ & $>99 \%$ \\
4 & 001900580010 & 1074.66973 & 1799 & 19.4 & 0.0106 & $2.47 \pm 0.17$ & $>99 \%$ \\
5 & 001900590010 & 1074.69176 & 1797 & 16.8 & 0.0111 & $2.34 \pm 0.14$ & $<70 \%$ \\
6 & 001900900010 & 1075.48207 & 1796 & 19.4 & 0.0282 & $3.46 \pm 0.16$ & $>99 \%$ \\
7 & 001900910010 & 1075.50407 & 1796 & 20.9 & 0.0287 & $3.28 \pm 0.18$ & $>99 \%$ \\
8 & 002000060010 & 1076.49525 & 3288 & 24.8 & 0.0502 & $3.38 \pm 0.12$ & $>99 \%$ \\
9 & 002000160010 & 1076.71562 & 1783 & 13.4 & 0.0550 & $1.20 \pm 0.16$ & $\sim 70 \%$ \\
10 & 002000260010 & 1076.93582 & 1773 & 23.7 & 0.0598 & $2.57 \pm 0.16$ & $\sim 99 \%$ \\
11 & 002000350010 & 1077.13415 & 1776 & 30.7 & 0.0641 & $3.26 \pm 0.13$ & $>99 \%$ \\
12 & 002000360010 & 1077.15594 & 1795 & 29.5 & 0.0646 & $3.53 \pm 0.18$ & $>99 \%$ \\
13 & 002000540010 & 1077.55208 & 1795 & 38.3 & 0.0732 & $4.58 \pm 0.18$ & $>99 \%$ \\
\hline
\end{tabular}

different patterns of observations to check instrument behaviour and imaging performances.

The JEM-X monitor consists of two identical codedaperture mask telescopes co-aligned with the other instruments onboard INTEGRAL. The detectors are microstrip gas chambers with a collecting area of $500 \mathrm{~cm}^{2}$ per unit located at a distance of $3.4 \mathrm{~m}$ from the coded mask. JEM-X was designed to work in the energy range $3-35 \mathrm{keV}$ with an energy resolution of $\Delta E / E=0.47 \times(E / 1 \mathrm{keV})^{-1 / 2}$ and a timing resolution of $122 \mu \mathrm{s}$. A detailed description of JEM-X can be found in Lund et al. (2003).

A gas gain decrease (Lund et al. 2003) modified the low energy threshold of the detectors. The $50 \%$ efficiency level is now reached at $4.2 \mathrm{keV}$, instead of the pre-launch value of $3.5 \mathrm{keV}$. Above $25 \mathrm{keV}$, the quantum efficiency is determined by the gradually diminishing absortion cross section of the Xenon gas (at $25 \mathrm{keV}$, the efficiency is $\sim 35 \%$ ). Therefore, for a relatively weak source like EXO 2030+375, the optimum energy range goes from 5 to $25 \mathrm{keV}$. Below $5 \mathrm{keV}$ the electronic noise dominates; above $25 \mathrm{keV}$, the background does.

An outburst of EXO 2030+375 took place during December 9 to 17, 2002 corresponding to INTEGRAL orbit revolutions 19 to 21 . During these revolutions INTEGRAL observed about 250 pointings in the Cygnus region, but in the majority of these EXO $2030+375$ is far from the JEM-X field of view. Only in about $10 \%$ of the pointings was the source inside the JEM-X fully coded field of view. Another constraint is the proximity of the two much stronger sources Cyg X-1 and Cyg X-3. In many pointings the signal from these sources increases the background for our source very significantly. As a result, we have found only 13 pointings in which the source was detected by JEM-X. These detections took place between December 9-13, 2002, which correspond to revolutions 19 and 20 and orbital phases from 0.988 to 0.0732 , according to the ephemeris given by Wilson et al. (2002). This phase interval corresponds approximately with the peak of the outburst. The 13 EXO 2030+375 detections are summarised in Table 1. We give the Science Window Identifier (SWID), the INTEGRAL Julian Date (IJD = JD-2 451544.5), the observing time, the signal-to-noise $(S / N)$, the orbital phase, the count rate obtained from the light curve extraction and the pulse period detection confidence level $(C L)$. Of these 13 detections, 3 were not considered due to a low signal to noise (Obs. 2) or instrumental problems that are under investigation (Obs. 9 and 10).

The data reduction was carried out with the Offline Scientific Analysis software, release 1.1, including those updates which were available up to 2002 June 20, whereas the data analysis was done using XSPEC and XRONOS packages. The latest response matrices (jmx1_rmf_grp_0004.fits and jmx2_rmf_grp_0004.fits) have been used.

\section{Results and discussion}

\subsection{Timing analysis}

Figure 1 displays the JEM-X light curve of the entire observing run ( 3.5 days) in the energy range 5 to $25 \mathrm{keV}$. The flux varies a factor of 2 throughout the observations. For the sake of comparison simultaneous RXTE, ISGRI observations and data from a previous outburst detected by BATSE (Norton et al. 1994) are also given. Note the shift of the outburst peak for Dec. 2002 observations with respect to the previous BATSE detection. This shift has been seen in other outbursts. It has been attributed to a global one-armed oscillation propagating in the Be star disc (Wilson et al. 2002).

For all those observations with $S / N$ greater than 19 , we can determinate the pulse period with a confidence level higher than $99 \%$. The average pulse period for those observations is $41.66 \pm 0.05 \mathrm{~s}$ and the average pulse fraction is $60 \%$. Figures 2 and 3 show the the time-averaged pulse profile and the power spectrum of the best detection on 12th of December 2002 (SWID $=00200540010$, Obs. 13). The straight line in Fig. 3 represents the $99 \%$ confidence level of detection for 1024 frequencies (van der Klis 1989). The power spectrum was obtained by performing a Fast Fourier Transform on the 1-s binned light curve of Obs. 13. The normalization is given by $2 / N_{\text {phot }}$, where $N_{\text {phot }}$ is the total number of photons per transform (Leahy et al. 1983; van der Klis 1989). 

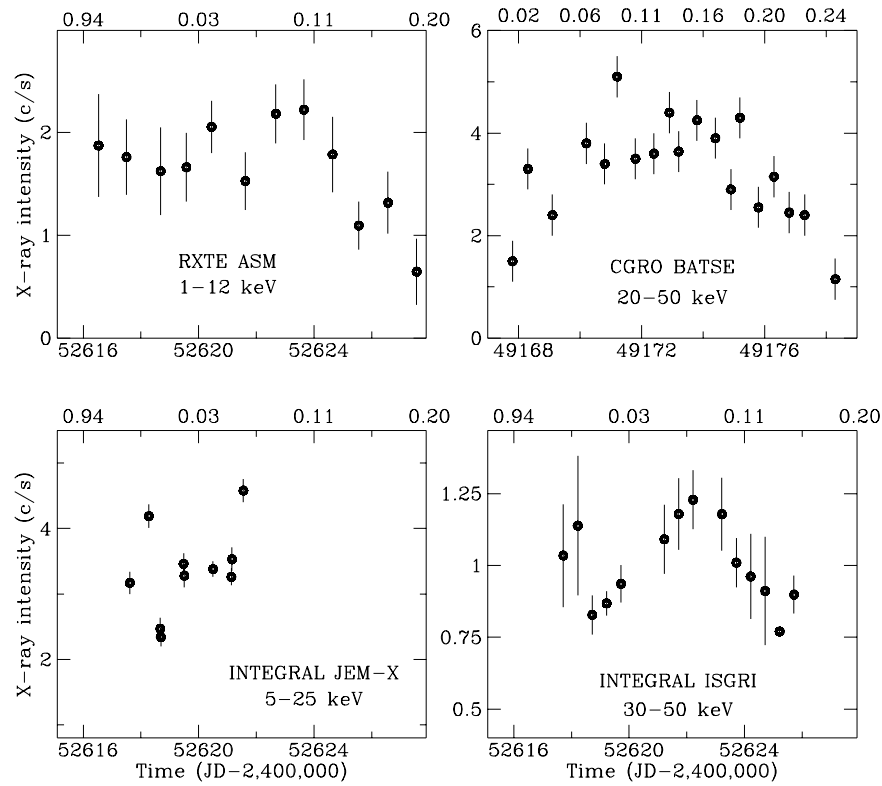

Fig. 1. Long-term light curve of EXO 2030+375 during the X-ray outburst. All light curves except that of CGRO BATSE (Norton et al. 1994) display simultaneous data. ISGRI data are normalized to the mean flux value.

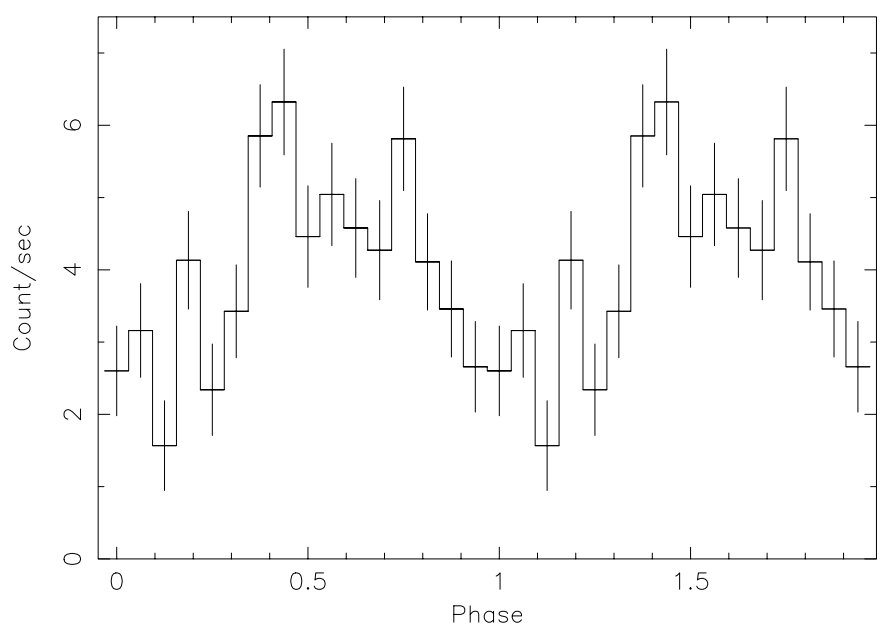

Fig. 2. EXO 2030+375 time-averaged pulse profile of the best detection, SWID = 002000540010 .

\subsection{Spectral analysis}

In order to look for spectral shape changes throughout the $\mathrm{X}$-ray outburst we first analysed all ten good JEM-X spectra separately. We tried different models to fit the energy spectra. Even though a single power law $(\Gamma \sim 2)$ or an exponential $\left(E_{\mathrm{c}} \sim 6 \mathrm{keV}\right)$ formally gave acceptable fits (reduced $\chi^{2}$ lower than 2), the residuals present a well defined structure, indicating that a second model component is needed.

The inclusion of a moderate amount of absorption $\left(N_{\mathrm{H}}=\right.$ $2-3 \times 10^{22} \mathrm{~cm}^{-2}$ ) does not improve the fit. As in the previous cases, the largest residuals occur below $\sim 9 \mathrm{keV}$, indicating that absorption does not affect the spectrum at these relatively high energies $(\sim 4-9 \mathrm{keV})$. If we let the column density be a free parameter we obtain $N_{\mathrm{H}} \approx 30 \times 10^{22} \mathrm{~cm}^{-2}$, that is more than an order of magnitude larger than previous X-ray and

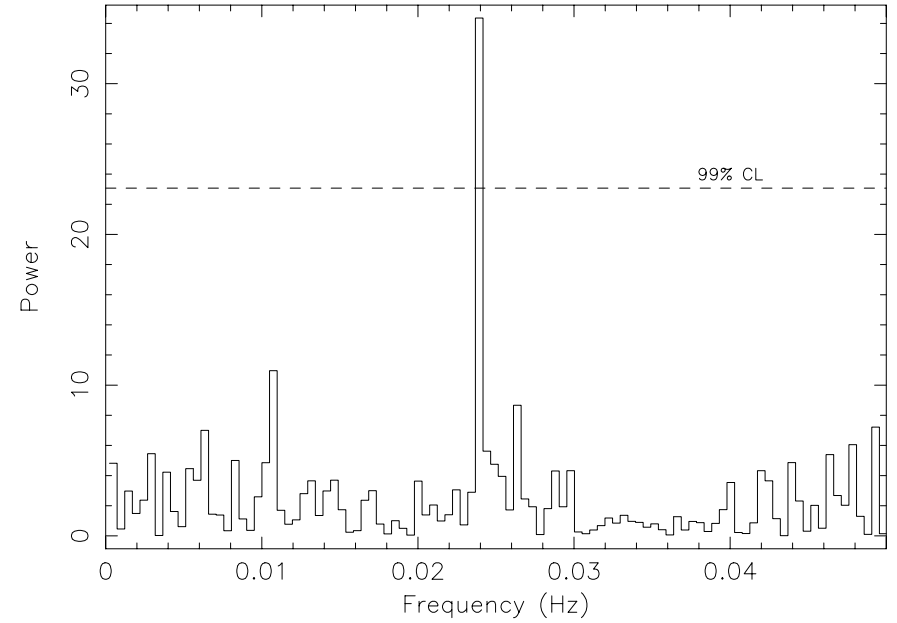

Fig. 3. EXO $2030+375$ power spetrum for 1024 frequencies. The dashed line represents the $99 \%$ confidence level.

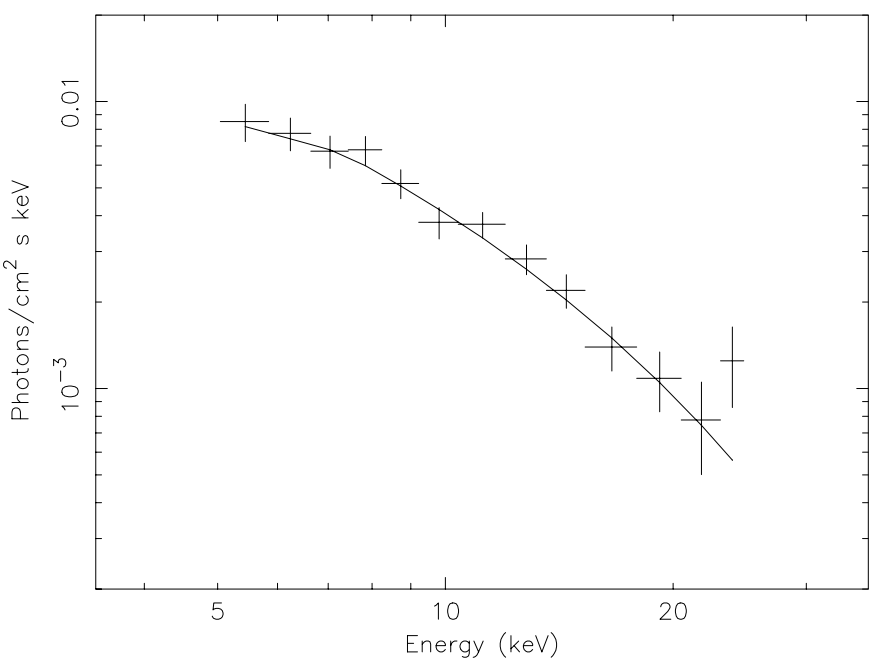

Fig. 4. Energy spectrum of observation $13($ SWID $=002000540010)$. The continuous line represents a powerlaw with a high energy cutoff.

optical observations, where the absorption was seen to vary in the range $2-3 \times 10^{22} \mathrm{~cm}^{-2}$ despite big changes in luminosity (Coe et al. 1988; Reynolds et al. 1993; Sun et al. 1994; Mavromatakis 1994; Reig \& Coe 1999). The detection of an iron line at $\sim 6.4 \mathrm{keV}$ with moderate intensity (equivalent width $\sim 200 \mathrm{eV}$ ) has been previously reported (Reynolds et al. 1993; Reig \& Coe 1999). The relatively low signal to noise of the observations and the modest energy resolution of JEM-X prevent us from detecting the line in the JEM-X spectrum.

Restricting the spectrum to energies in the range 5-25 keV, the continuum can be fit with the canonical model for accreting pulsars, namely, a power law with an exponential cutoff at high energies. No significant differences are found among the different spectra. Figure 4 shows a representative energy spectrum. The average spectral parameters are given in Table 2 . The photon index and cutoff energy agree with previous results (Reig \& Coe 1999).

If each individual spectrum is fitted using a single powerlaw model in the energy range $9-25 \mathrm{keV}$, some marginal differences are found. Figure 5 shows the photon spectral index 


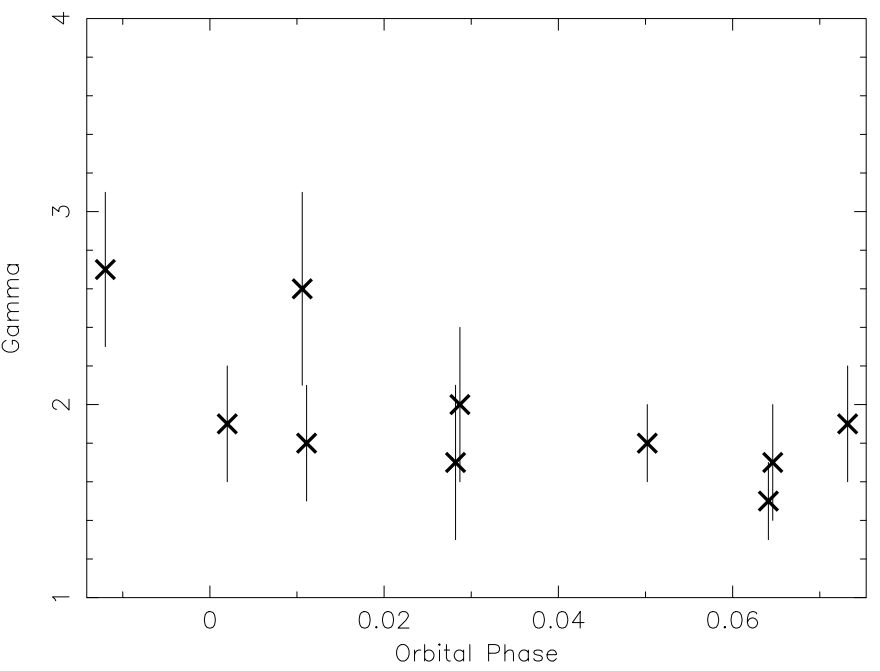

Fig. 5. Photon spectral index of the power law model in the energy range 9-25 versus orbital phase. Error bars are $1 \sigma$ confidence region.

Table 2. Results of the spectral fit. Errors are within $90 \%$ of confidence level.

\begin{tabular}{lc}
\hline \hline Parameter & Value \\
\hline \hline$\Gamma$ & $0.52 \pm 0.12$ \\
$E_{\mathrm{c}}(\mathrm{keV})$ & $7.8 \pm 1.4$ \\
$E_{\text {fold }}(\mathrm{keV})$ & 10.0 (fixed) \\
Flux $^{a}(5-25 \mathrm{keV})$ & 7.3 \\
$\chi_{\text {red }}^{2}$ & 1.46 \\
\hline${ }^{a}$ in units of $10^{-10} \mathrm{erg} \mathrm{cm}^{-2} \mathrm{~s}^{-1}$
\end{tabular}

as a function of the orbital phase. At periastron passage the source shows a slightly softer spectrum than later in the orbit. The average value of the photon index for the first four observations is $2.4 \pm 0.3$, compared to $1.7 \pm 0.2$ at orbital phase larger than 0.02 . This result is supported by the timing ISGRI and RXTE simultaneous data. As it can be seen in Fig. 1, the ISGRI light curve presents a maximum at 0.07 orbital phase, whereas no changes are seen in the RXTE data at the same phase.

The average X-ray luminosity in the energy range 5$25 \mathrm{keV}$ was $2.1 \times 10^{36} \mathrm{erg} \mathrm{s}^{-1}$ for an assumed distance of $5 \mathrm{kpc}$ (Parmar et al. 1989). This value of the luminosity is of the same order as previous type I outbursts of this source. Reig \& Coe (1999) found that the X-ray luminosity changed from $\sim 9 \times 10^{35}$ to $\sim 4 \times 10^{36} \mathrm{erg} \mathrm{s}^{-1}$ for a variation in orbital phase from 0.86 to 0.08 in the energy range 2.7 to $30 \mathrm{keV}$. This value is typical of $\mathrm{Be} / \mathrm{X}$-ray binaries during type I outbursts. The LS992/RX J0812.43114 is similar in this respect to EXO 2030+375.
LS992 shows 80-day periodic outbursts and X-ray pulsations with a pulse period of 31.88 s (Reig \& Roche 1999; Corbet \& Peele 2000) and variations in luminosity of $2-4 \times 10^{36} \mathrm{erg} \mathrm{s}^{-1}$ (Corbet \& Peele 2000).

\section{Conclusion}

The sensitivity of JEM-X allowed us to perform an $\mathrm{X}$ ray timing and spectral analysis of the $\mathrm{Be} / \mathrm{X}$-ray binary EXO 2030+375 during an X-ray outburst. X-ray pulsations with a pulse period of $46.66 \pm 0.05 \mathrm{~s}$ are clearly detected throughout the outburst. The average $\mathrm{X}$-ray luminosity during the observations was $2.1 \times 10^{36} \mathrm{erg} \mathrm{s}^{-1}$ in the energy range $5-25 \mathrm{keV}$. The results reported in this work confirms the performance requirements of JEM-X.

Acknowledgements. We are grateful to SDAST and DSRI JEM-X team for providing us with the data and their support and advice in the development of this work.

\section{References}

Balona, L. A. 2000, The Be Phenomenon in Early-Type Stars, ASP Conf. Ser., 214, 1

Coe, M. J., Longmore, A., Payne, B. J., \& Hanson, C. G. 1988, MNRAS, 232, 865

Coe, M. J. 2000, The Be Phenomenon in Early-Type Stars, ASP Conf. Ser., 214, 656

Corbet, R. H. D., \& Peele, A. G. 2000, ApJ, 530, L33, ApJS

Leahy, D. A., Darbro, W., Elsner, R. F., et al. 1983, ApJ, 266, 160

Lund, N., Brandt, S., Budtz-Joergensen C., et al. 2003, A\&A, 411, $\mathrm{L} 231$

Mavromatakis, F. 1994, A\&A, 285, 209

Motch, C., \& Janot-Pacheco, E. 1987, A\&A, 182, L55

Norton, A. J., Chakrabarty, D., Coe, M. J., et al. 1994, MNRAS, 271, 981

Parmar, A. N, White, N. E., Stella, L., Izzo, C., \& Ferri, P. 1989, ApJ, 338,359

Reig, P., \& Coe, M. J. 1998, MNRAS, 294, 118

Reig, P., Stevens, J. B., Coe, M. J., \& Fabregat, J. 1998, MNRAS, 301, 42

Reig, P., \& Coe, M. J. 1999, MNRAS, 302, 700

Reig, P., \& Roche, P. 1999, MNRAS, 306, 95

Reynolds, A. P, Parmar, A. N, \& White, N. E. 1993, ApJ, 414, 302

Sun, X. J., Li, T. P., Wu, M., \& Cheng, L. X. 1994, A\&A, 289, 127

van der Klis 1989, Proc. NATO Advanced Study Institute Timing Neutron Stars

Wilson, C. A., Mark, H. F., Coe, M. J., Laycock, S., \& Fabregat, J. 2002, ApJ, 570, 287 